Article

\title{
Broadened Angle-Insensitive Near-Perfect Absorber Based on Mie Resonances in Amorphous Silicon Metasurface
}

\author{
Jiangnan Si, Shuang Liu, Weiji Yang, Xuanyi Yu, Jialin Zhang and Xiaoxu Deng * \\ State Key Laboratory of Advanced Optical Communication Systems and Networks, Key Laboratory for Laser \\ Plasmas (Ministry of Education), School of Physics and Astronomy, Shanghai Jiao Tong University, \\ Shanghai 200240, China; 1359231351370@sjtu.edu.cn (J.S.); 503313461@sjtu.edu.cn (S.L.); \\ yangweiji@sjtu.edu.cn (W.Y.); yxy1593725@sjtu.edu.cn (X.Y.); dorismalfoy@sjtu.edu.cn (J.Z.) \\ * Correspondence: xxdeng@sjtu.edu.cn
}

Received: 3 August 2020; Accepted: 24 August 2020; Published: 1 September 2020

\begin{abstract}
A broadband near-perfect absorber is analyzed by an amorphous silicon (a-Si) hook shaped nanostructure metasurface. The transmission and reflection coefficients of the metasurface are investigated in the point electric and magnetic dipole approximation. By combining square and semicircle nanostructures, the effective polarizabilities of the a-Si metasurface calculated based on discrete dipole approximation (DDA) exhibit broadened peaks of electric dipole (ED) and magnetic dipole (MD) Mie resonances. The optical spectra of the metasurface are simulated with different periods, in which suppressed transmission are shifted spectrally to overlap with each other, leading to broadened enhanced absorption induced by interference of ED and MD Mie resonances. The angle insensitive absorption of the metasurface arrives $95 \%$ in simulation and $85 \%$ in experiment in spectral range from $564 \mathrm{~nm}$ to $584 \mathrm{~nm}$, which provides potential applicability in nano-photonic fields of energy harvesting and energy collection.
\end{abstract}

Keywords: amorphous silicon metasurface; perfect absorption; Mie resonances

\section{Introduction}

Metasurfaces are artificial planar material composed of spatially arranged subwavelength structures, which exhibit remarkable flexibility in manipulating the properties of light at an optically thin interface [1-3]. Perfect absorbers based on metasurfaces for energy collection and accumulation have been widely researched, which are promising in applications of light harvesting [4-8], optical isolation [9,10] and so on. Dielectric nanostructure metasurfaces, holding advantages including nonradiative loss [11-13], resonant enhancement of both electric and magnetic field [14-19], attract much attention in research of high absorption induced by the Mie dipole resonances [20-23]. Recently, Länk's group has proposed a silicon nano-pillar metasurface in a total internal reflection geometry to realize near-perfect absorption in near-infrared region resulting from interference between coherent electric and magnetic dipoles scattering as well as reflection from supporting glass substrate [15]. Yang's group has reported a narrow band perfect absorber with a high quality factor by an amorphous silicon (a-Si) nano antenna metasurface, which is realized due to not only the coherent ED and MD Mie resonances but also the intrinsic loss of material [23]. Liu's group has put forward a germanium moth-eye nanostructure to obtain a perfect selective absorption attributed by the electric field enhancement due to electric and magnetic multipole resonances, which is insensitive to incident angle from $0^{\circ}$ to $75^{\circ}$ [24].

In this manuscript, a dielectric broadband near-perfect absorber is numerically studied and experimentally analyzed based on an amorphous silicon (a-Si) hook shaped nanostructure metasurface. The transmission and reflection coefficients of the a-Si hook shaped nanostructure metasurface are 
investigated by far-field scattering of electric and magnetic dipole moments. The electric dipole (ED) and magnetic dipole (MD) effective polarizabilities of the a-Si nanostructure metasurface are numerically calculated from simulated displacement currents based on the discrete dipole approximation (DDA). ED and MD Mie resonances of the a-Si metasurface, which induce suppression band in the simulated transmission spectra, are periodical adjustable in spectral position. Due to the interference between overlapped Mie resonances, the absorption spectrum reaches the maximum of $95 \%$ in a broadened spectral range from $564 \mathrm{~nm}$ to $584 \mathrm{~nm}$, which is induced by enhanced E-fields in different positions of the hook shaped nanostructure. Furthermore, the near perfect absorption is changeless with the incident angle varying from $0^{\circ}$ to $20^{\circ}$ due to angle insensitive Mie resonances. The nanoscale a-Si metasurface was fabricated and the absorption spectrum measured by a convergent spectrometer arrives $85 \%$ in the same resonant spectral range in simulation. The fabricated broadened band incident-angle-insensitive near-perfect absorber has promising prospects of practical nano-photonic applications in area of optical trapping, energy accumulation and energy harvesting.

\section{Materials and Methods}

The incident-angle insensitive dielectric broadband near-perfect absorber in this manuscript is an a-Si hook shaped nanostructure periodical array metasurface, of which the schematic is presented in Figure 1a. The unit cell of the metasurface contains a nano square and a nano semicircle, as shown in the inset of Figure 1a. The edge width of a square is $w=70 \mathrm{~nm}$. The radius of a semicircle is $r=75 \mathrm{~nm}$. Periods of the nanostructure metasurface in the $\mathrm{x}$ and y direction are $P_{x}=220 \mathrm{~nm}$ and $P_{y}=340 \mathrm{~nm}$, respectively. The a-Si nanostructure thickness $h$ is $115 \mathrm{~nm}$. The hook shaped nanostructure metasurface is on the top of $\mathrm{SiO}_{2}$ substrate and submerged in immersion oil. The refractive indexes of $\mathrm{SiO}_{2}$ and immersion oil are $n_{\mathrm{SiO}_{2}}=1.528$ and $n_{\mathrm{Oil}}=1.485$, respectively. The three-dimensional finite-difference time-domain method (FDTD) is utilized to simulate the optical spectra and the electric fields of the a-Si metasurface. The boundary condition in FDTD simulation is periodic in the $x$ and $y$-directions, and the perfect matched layer (PML) in the z-direction. A plane wave is set in the FDTD simulation, of which the wave vector is along the negative $\mathrm{z}$ direction, the electric field is parallel to the $\mathrm{x}$ direction and the magnetic field is parallel to the y direction. The reflection $(R)$, transmission $(T)$ and the absorption $(A)$ of the a-Si metasurface and the displacement currents of the a-Si hook shaped nanostructure are simulated by FDTD.

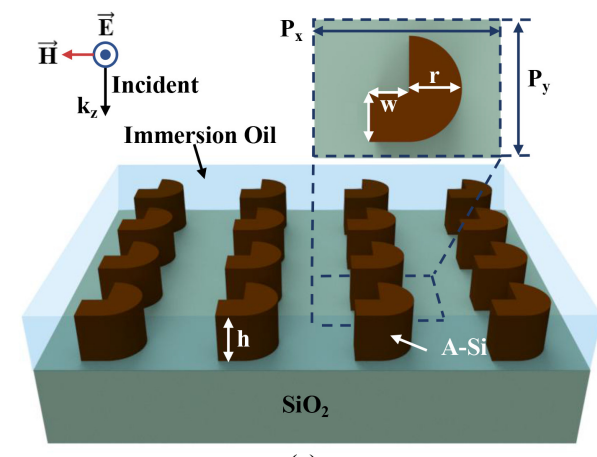

(a)

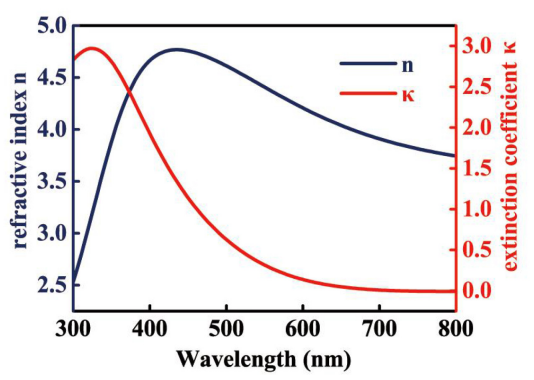

(b)

Figure 1. (a) The schematic of the a-Si hook shaped nanostructure metasurface. The unit cell is shown in the top figure. (b) Refractive index $n$ (blue curve) and extinction coefficient $\kappa$ (red curve) of amorphous silicon film.

In the fabrication process, the a-Si film was deposited on the $\mathrm{SiO}_{2}$ substrate by Plasma Enhanced Chemical Vapor Deposition system. The complex refractive index of the a-Si is $n_{a-S i}=n+i \kappa$, where the real part is defined as the refractive index $n$ and the imaginary part is defined as the extinction coefficient $\kappa . n$ and $\kappa$ are measured by spectroscopic ellipsometry apparatus from the fabricated a-Si film, as presented in Figure 1b. Photoresist was first coated on the a-Si film, then $20 \mathrm{~nm}$ thick aluminum 
film was deposited on the photoresist as a conducting layer. The a-Si hook shaped nanostructure metasurface was fabricated by electron-beam lithography (EBL) and the silicon etching system. Finally, the immersion oil ( $n=1.485$ ) was added from the edge of the fabricated metasurface and submerge the nanostructures.

\section{Results and Discussion}

\subsection{Numerical and Simulated Results}

The broadband Mie resonances of the a-Si hook shaped nanostructure metasurface are investigated by effective polarizabilities based on discrete dipole approximation (DDA). The light-induced electric dipole (ED) and magnetic dipole (MD) moment of an a-Si hook shaped nanostructure in the periodical array are related with displacement currents [25]:

$$
p=-\frac{1}{i \omega} \int J d^{3} r ; \quad m=\int(r \times J) d^{3} r
$$

where $\omega$ is the frequency of incident light, $J$ is the displacement current. Under illumination of x-polarized light, only the ED moment $p_{x}$ and the MD moment $m_{y}$ of the a-Si hook shaped nanostructure contribute to the light scattering in the far-field approach due to the periodicity of the metasurface [26], while the scattered light contributed by $m_{z}$ is ignored. Every a-Si hook shaped nanostructure are seen as point sources with same effective ED polarizability $\alpha_{e f f}^{E}=p_{x} / \varepsilon_{0} E^{0}$ and MD polarizability $\alpha_{e f f}^{M}=m_{y} / H^{0}$, where $E^{0}$ and $H^{0}$ are the electric field and magnetic field of the x-polarized light illuminated on the periodical array, respectively, and $\varepsilon_{0}$ is dielectric constant of vacuum. Based on the scattered fields generated by the point source dipole moments of the a-Si hook shaped nanostructure in the far-field approach, the transmission $(t)$ and reflection $(r)$ coefficients of the periodical array are:

$$
t=1+\frac{i k_{0}}{2 S_{L}}\left(\alpha_{e f f}^{E}+\alpha_{e f f}^{H}\right), \quad r=\frac{i k_{0}}{2 S_{L}}\left(\alpha_{e f f}^{E}-\alpha_{e f f}^{H}\right)
$$

$k_{0}$ is the wave number; $S_{L}$ is the unit cell area. According to Equation (2), the transmission and reflection coefficients simultaneously approach zero when:

$$
\operatorname{Re}\left(\alpha_{e f f}^{E}\right)=\operatorname{Re}\left(\alpha_{e f f}^{H}\right)=0, \quad \operatorname{Im}\left(\alpha_{e f f}^{E}\right)=\operatorname{Im}\left(\alpha_{e f f}^{H}\right)=S_{L} / k_{0}
$$

The effective polarizabilities of the square only nanostructure, the semicircle only nanostructure and the hook shaped nanostructure metasurfaces are calculated by utilizing FDTD-simulated displacement currents in spectral range from $400 \mathrm{~nm}$ to $800 \mathrm{~nm}$ with $P_{x}=220 \mathrm{~nm}$ and $P_{y}=340 \mathrm{~nm}$ based on DDA, as shown in Figure 2. The ED and MD effective polarizabilities of the square only nanostructure metasurface are shown by the red and blue curves in Figure $2 \mathrm{a}$, respectively. $\operatorname{Im}\left(\alpha_{e f f}^{E}\right)$ has a sharp peak and $\operatorname{Im}\left(\alpha_{e f f}^{M}\right)$ possesses a low peak on behalf of the ED and MD resonances, respectively, which are at spectral positions of $540 \mathrm{~nm}$ and $500 \mathrm{~nm}$ where the corresponding real parts of effective polarizabilties are around zero. For the semicircle only nanostructure metasurface, $\operatorname{Im}\left(\alpha_{e f f}^{E}\right)$ and $\operatorname{Im}\left(\alpha_{e f f}^{M}\right)$ hold peaks with different value at spectral positions of $580 \mathrm{~nm}$ and $570 \mathrm{~nm}$, respectively, as shown by red and blue dashed curves in Figure 2b. With respect to the hook shaped nanostructure metasurface, $\operatorname{Im}\left(\alpha_{\text {eff }}^{E}\right)$ and $\operatorname{Im}\left(\alpha_{e f f}^{M}\right)$ both have a broadened peak approximately equal to $S_{L} / k_{0}$ in spectral range from $564 \mathrm{~nm}$ to $584 \mathrm{~nm}$, meanwhile $\operatorname{Re}\left(\alpha_{e f f}^{E}\right)$ and $\operatorname{Re}\left(\alpha_{e f f}^{M}\right)$ are around zero, as shown in Figure 2c. The ED and MD Mie resonances of the metasurface are both broadened due to the employed square and semicircle in a hook shaped nanostructure. 

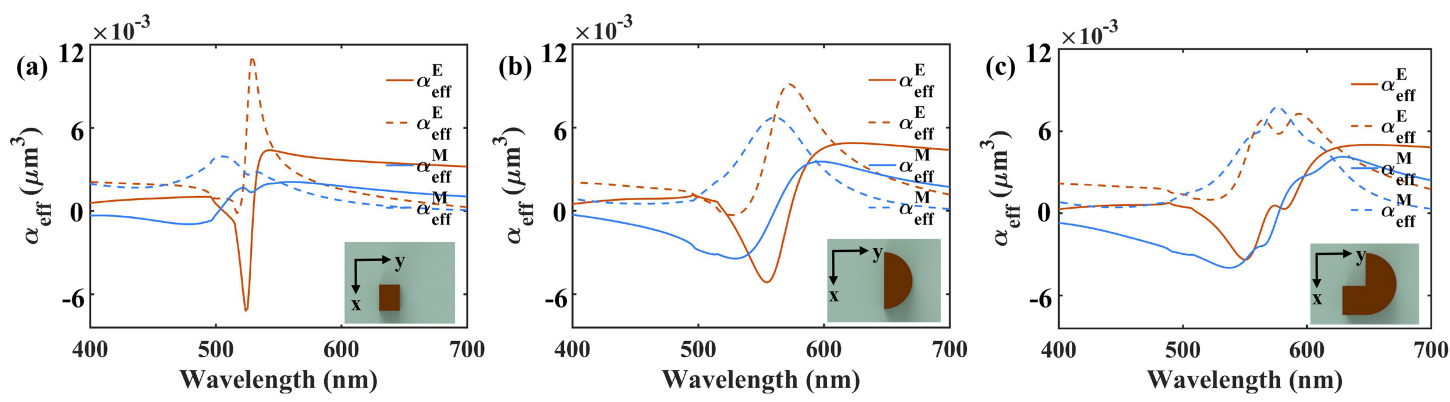

Figure 2. The effective polarizabilities of the square only nanostructure (a), the semicircle only nanostructure (b) and the hook shaped nanostructure (c) with periods $P_{x}=220 \mathrm{~nm}, P_{y}=340 \mathrm{~nm}$.

The impact of period on optical spectra of the a-Si hook shaped nanostructure metasurface is simulated by FDTD. The simulated transmission $(T)$, reflectance $(R)$ and absorption $(A)$ spectra of the a-Si hook shaped nanostructure metasurface with varying period $P_{x}$ and fixed $P_{y}=340 \mathrm{~nm}$ are shown in Figure $3 \mathrm{a}-\mathrm{c}$, respectively. Two broadened suppressed transmission, one red shifted with $P_{x}$, the other fixed around $570 \mathrm{~nm}$ exist in Figure 3a, which are induced by MD and ED resonances, respectively. When $P_{x} \approx 220 \mathrm{~nm}$, in spectral range from $564 \mathrm{~nm}$ to $584 \mathrm{~nm}$, reflection is strongly suppressed, meanwhile absorption approaches the maximum of $95 \%$, as presented in Figure $3 b, c$, respectively. The interference of overlapped ED and MD resonances results in suppressed transmission by the electric field localization and suppressed reflection by the Kerker effect, therefore the broadband absorption enhancement of the a-Si hook shaped nanostructure metasurface is achieved.
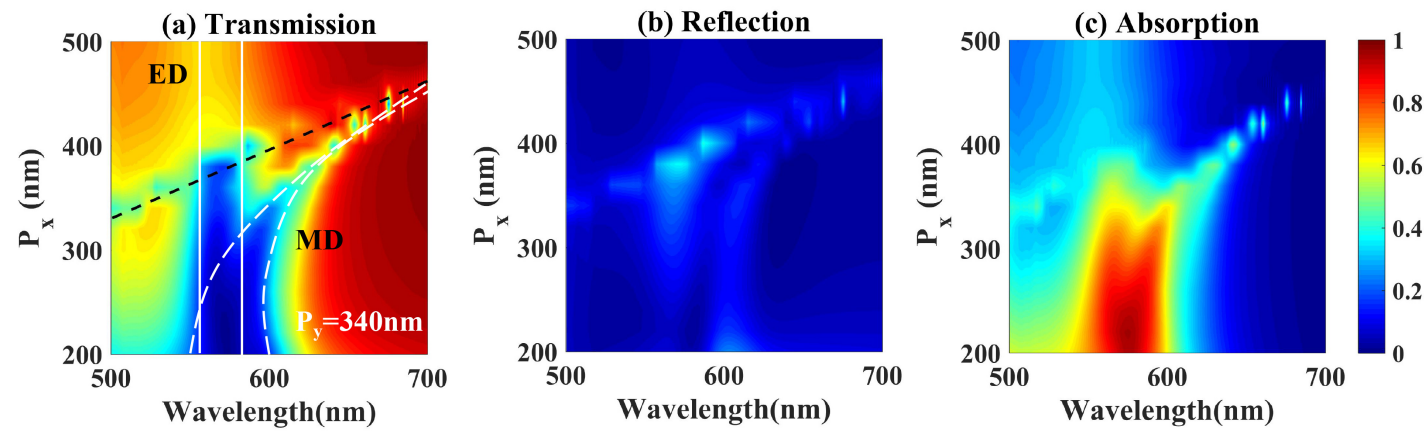

Figure 3. The simulated (a) transmission, (b) reflection, and (c) absorption spectra of the metasurface with different periods $P_{x}$. $P_{y}$ is fixed at $340 \mathrm{~nm}$.

The electric field (E-field) distributions inside the a-Si hook shaped nanostructure metasurface at different wavelengths are simulated and analyzed. The E-field distributions of the hook shaped nanostructure in the $x-y$ plane in the overlapped spectral range of ED and MD resonances from $564 \mathrm{~nm}$ to $584 \mathrm{~nm}$ are more intensive compared with those at non-resonant wavelengths of $504 \mathrm{~nm}$ and $654 \mathrm{~nm}$, as presented in Figure 4. The enhancement of E-field distributions is induced in the square and semicircle nanostructure separately at different wavelengths, leading to the strengthened absorption in a broadened spectral range.

The optical spectra of the a-Si hook-shaped nanostructure metasurface are simulated with different incident angle $\theta$. The simulated reflection, transmission and absorption spectra with incident angle $\theta$ from $0^{\circ}$ to $50^{\circ}$ in spectral range from $500 \mathrm{~nm}$ to $700 \mathrm{~nm}$ are presented in Figure $5 \mathrm{a}-\mathrm{c}$, respectively. The suppression band and suppression degree of both reflection and transmission are not affected by the incident angle increasing from $0^{\circ}$ to $20^{\circ}$, therefore the enhanced absorption is unchanged in both spectral region and maximum value. When the incident angle increases from $20^{\circ}$ to $50^{\circ}$, the suppression band of reflection is narrowed down, and the suppressed transmission is broadened and increased less than 3\%, therefore the enhanced absorption of the metasurface is broadened and 
declined below 3\%. The near-perfect absorption of the a-Si hook shaped nanostructure metasurface is efficient with incident angle varing from $0^{\circ}$ to $20^{\circ}$ due to incident angle insensitive ED and MD Mie resonances.

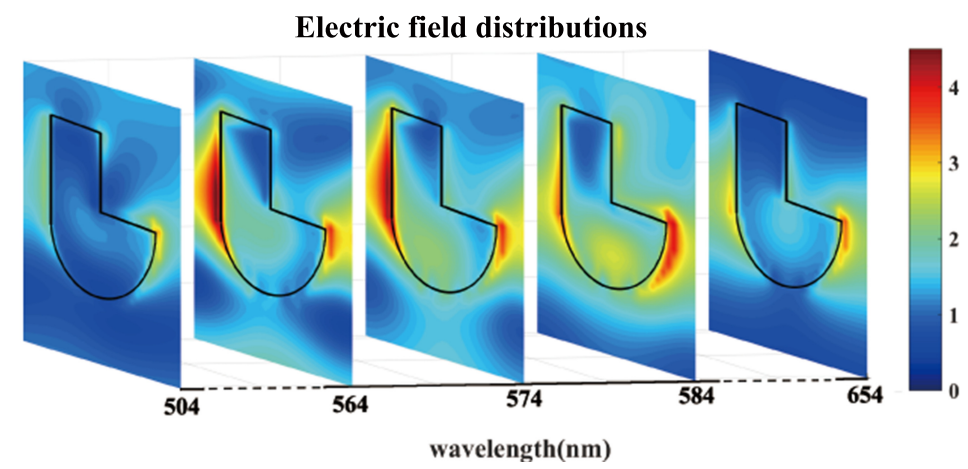

Figure 4. The electric field (E-field) distributions of the a-Si hook shaped nanostructure in the $x-y$ plane when electric dipole (ED) and magnetic dipole (MD) resonances are overlapped at wavelength of $504 \mathrm{~nm}, 564 \mathrm{~nm}, 574 \mathrm{~nm}, 584 \mathrm{~nm}$ and $654 \mathrm{~nm}$.
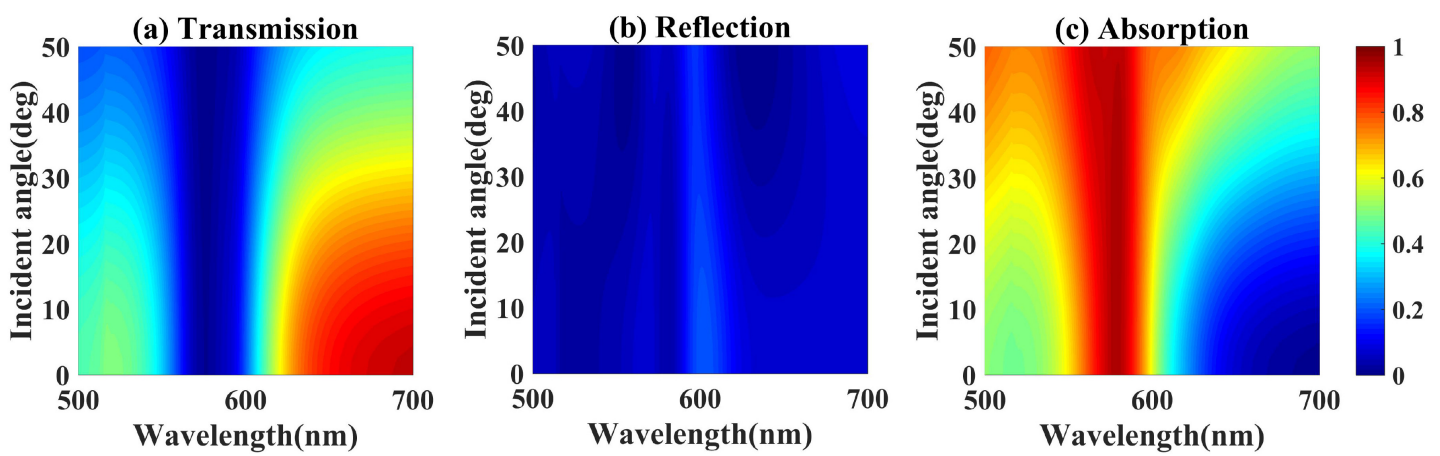

Figure 5. The simulated (a) reflection, (b) transmission and (c) absorption spectra of the a-Si nanostructure metasurface as a function of incident angle.

\subsection{Experimental Results}

The scanning electron microscopy (SEM) image of the fabricated a-Si hook shaped nanostructure metasurface is presented in Figure $6 \mathrm{a}$, in which the radius of a semicircle is $r \approx 77 \mathrm{~nm}$ and the edge length of the square is $w \approx 72 \mathrm{~nm}$. Periods of the fabricated metasurface are $P_{x} \approx 217 \mathrm{~nm}, P_{y} \approx 340 \mathrm{~nm}$. Total pattern area of the fabricated metasurface is $499.4 \mu \mathrm{m} \times 499.8 \mu \mathrm{m}$. The thickness of the fabricated a-Si film was $114.8 \mathrm{~nm}$. Transmission $(T)$, reflection $(R)$ and absorption $(A)$ spectra of the fabricated a-Si metasurface were measured by a convergent spectrometer, as presented by the green, blue and red solid curves in Figure 6b, respectively, which exhibit same trend as simulated results shown by the dashed curves. The near-perfect absorption in experiment reaches 85\% in the spectral range from $564 \mathrm{~nm}$ to $584 \mathrm{~nm}$, which declines $10 \%$ compared with simulated result. The reduction of the measured near-perfect absorption is caused by the nano fabrication deviation of shape of the a-Si nanostructures, while the impact of convergent spectrometer on the measured spectra is neglected due to the incident angle insensitivity of the a-Si metasurface. The metasurface is not completely submerged by the immersion oil, leading to residual air gaps between the hook shaped nanostructures which also results in the reduction of measured near-perfect absorption. 
(a)

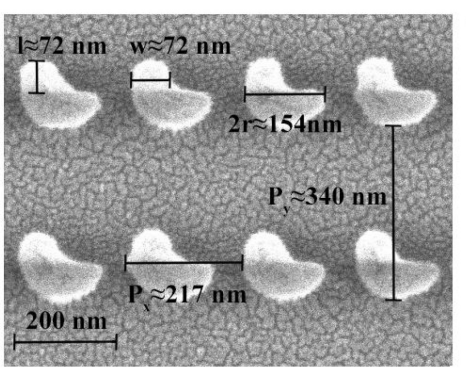

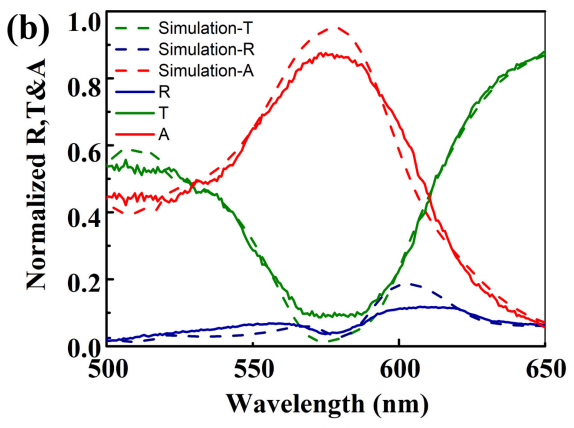

Figure 6. (a) The scanning electron microscopy (SEM) image of fabricated a-Si hook shaped nanostructure metasurface. (b) The measured reflection ( $R$, blu solid curve), transmission (T, green solid curve) and absorption $(A$, red solid curve) spectra of the fabricated metasurface. The simulated reflection, transmission and absorption of the metasurface are presented by the dashed curves.

\section{Conclusions}

In conclusion, a dielectric broadband near-perfect absorber based on amorphous silicon hook shaped nanostructure metasurface is investigated numerically and experimentally. The transmission and reflection coefficients of the a-Si metasurface are analyzed by far-field scattering of electric and magnetic dipole moments. By researching effective polarizabilities numerically calculated based on DDA, ED and MD Mie resonances of the a-Si metasurface are broadened in spectrum due to the employed hook shaped nanostructure. Angle insensitive near-perfect absorption of the a-Si metasurface reaches $95 \%$ in simulation and $85 \%$ in experiment in a broadened spectral range from $564 \mathrm{~nm}$ to $584 \mathrm{~nm}$ by the interference of Mie resonances. The proposed a-Si hook shaped nanostructure metasurface with characteristics of broadened bandwidth and insensitivity of incident angle, is prospective in practical applications including optical isolation device and energy harvesting system.

Author Contributions: Software, J.S., J.Z. and X.Y.; validation, W.Y.; formal analysis, J.S., S.L., and W.Y.; data curation, J.S.; writing - original draft preparation, J.S.; writing-review and editing, X.D.; funding acquisition, X.D. All authors have read and agreed to the published version of the manuscript.

Funding: This research was funded by National Natural Science Foundation of China, grant numbers 61675131 and 61927822.

Acknowledgments: The Center of Advanced Electronic Materials and Devices (AEMD) of Shanghai Jiao Tong University support us to fabricate the nanoscale amorphous silicon metasurfaces.

Conflicts of Interest: The authors declare no conflict of interest.

\section{References}

1. Zhao, Q.; Zhou, J.; Zhang, F.; Lippens, D. Mie resonance-based dielectric metamaterials. Mater. Today 2009, 12, 60-69. [CrossRef]

2. Chen, S.; Li, Z.; Zhang, Y.; Cheng, H.; Tian, J. Phase Manipulation of Electromagnetic Waves with Metasurfaces and Its Applications in Nanophotonics. Adv. Opt. Mater. 2018, 6, 1800104. [CrossRef]

3. Chang, S.; Guo, X.; Ni, X. Optical Metasurfaces: Progress and Applications. Annu. Rev. Mater. Res. 2018, 48, 279-302. [CrossRef]

4. Zheng, X.; Xiao, Z.; Ling, X. Broadband visible perfect absorber for sensor based on ultra-thin metamaterial. J. Mater. Sci. Mater. Electron. 2017, 28, 7739-7744. [CrossRef]

5. Magdi, S.; El-Diwany, F.; Swillam, M.A. Broadband MIR harvester using silicon nanostructures. Sci. Rep. 2019, 9, 5829. [CrossRef] [PubMed]

6. Desouky, M.; Mahmoud, A.M.; Swillam, M.A. An all silicon-based metamaterial for mid-IR energy harvesting. In Proceedings of the Photonic and Phononic Properties of Engineered Nanostructures VIII, San Francisco, CA, USA, 27 January-1 February 2018; Adibi, A., Lin, S.Y., Scherer, A., Eds.; International Society for Optics and Photonics: San Diego, CA, USA; 2018; Volume 10541, pp. 16-21. [CrossRef] 
7. Vismara, R.; Länk, N.O.; Verre, R.; Käll, M.; Isabella, O.; Zeman, M. Solar harvesting based on perfect absorbing all-dielectric nanoresonators on a mirror. Opt. Express 2019, 27, A967. [CrossRef]

8. Zhao, X.; Wang, Y.; Schalch, J.; Duan, G.; Cremin, K.; Zhang, J.; Chen, C.; Averitt, R.D.; Zhang, X. Optically Tunable All-Dielectric Broadband Terahertz Metamaterial Perfect Absorber. In Proceedings of the 2019 44th International Conference on Infrared, Millimeter, and Terahertz Waves (IRMMW-THz), Paris, France, 1-6 September 2019; pp. 1-3. [CrossRef]

9. Bosio, N.; Šípová Jungová, H.; Länk, N.O.; Antosiewicz, T.J.; Verre, R.; Käl, M. Plasmonic versus all-dielectric nanoantennas for refractometric sensing: A direct comparison. ACS Photonics 2019, 6, 1556-1564. [CrossRef]

10. Bezares, F.J.; Long, J.P.; Glembocki, O.J.; Guo, J.; Rendell, R.W.; Kasica, R.; Shirey, L.; Owrutsky, J.C.; Caldwell, J.D. Mie resonance-enhanced light absorption in periodic silicon nanopillar arrays. Opt. Express 2013, 21, 27587. [CrossRef]

11. Kuznetsov, A.I.; Miroshnichenko, A.E.; Brongersma, M.L.; Kivshar, Y.S.; Luk'yanchuk, B. Optically resonant dielectric nanostructures. Science 2016, 354, aag2472. [CrossRef]

12. Kruk, S.; Kivshar, Y. Functional Meta-Optics and Nanophotonics Governed by Mie Resonances. ACS Photonics 2017, 4, 2638-2649. [CrossRef]

13. Kivshar, Y.; Miroshnichenko, A. Meta-Optics with Mie Resonances. Opt. Photonics News 2017, $28,24$. [CrossRef]

14. Ra'di, Y.; Asadchy, V.S.; Kosulnikov, S.U.; Omelyanovich, M.M.; Morits, D.; Osipov, A.V.; Simovski, C.R.; Tretyakov, S.A. Full Light Absorption in Single Arrays of Spherical Nanoparticles. ACS Photonics 2015, 2, 653-660. [CrossRef]

15. Länk, N.O.; Verre, R.; Johansson, P.; Käll, M. Large-Scale Silicon Nanophotonic Metasurfaces with Polarization Independent Near-Perfect Absorption. Nano Lett. 2017, 17, 3054-3060. [CrossRef]

16. Babicheva, V.E.; Moloney, J.V. Lattice effect influence on the electric and magnetic dipole resonance overlap in a disk array. Nanophotonics 2018, 7, 1663-1668. [CrossRef]

17. Forouzmand, A.; Mosallaei, H. All-Dielectric C-Shaped Nanoantennas for Light Manipulation: Tailoring Both Magnetic and Electric Resonances to the Desire. Adv. Opt. Mater. 2017, 5, 1700147. [CrossRef]

18. Sun, S.; Zhou, Z.; Zhang, C.; Gao, Y.; Duan, Z.; Xiao, S.; Song, Q. All-Dielectric Full-Color Printing with $\mathrm{TiO}_{2}$ Metasurfaces. ACS Nano 2017, 11, 4445-4452. [CrossRef] [PubMed]

19. Paniagua-Dominguez, R.; Ha, S.T.; Kuznetsov, A.I. Active and Tunable Nanophotonics With Dielectric Nanoantennas. Proc. IEEE 2020, 108, 749-771. [CrossRef]

20. Alaee, R.; Albooyeh, M.; Rockstuhl, C. Theory of metasurface based perfect absorbers. J. Phys. D Appl. Phys. 2017, 50, 503002. [CrossRef]

21. Babicheva, V.E.; Evlyukhin, A.B. Resonant Lattice Kerker Effect in Metasurfaces with Electric and Magnetic Optical Responses. Laser Photonics Rev. 2017, 11, 1700132. [CrossRef]

22. Wang, Z.Y.; Zhang, R.J.; Wang, S.Y.; Lu, M.; Chen, X.; Zheng, Y.X.; Chen, L.Y.; Ye, Z.; Wang, C.Z.; Ho, K.M. Broadband optical absorption by tunable Mie resonances in silicon nanocone arrays. Sci. Rep. 2015, 5, 7810. [CrossRef]

23. Yang, C.Y.; Yang, J.H.; Yang, Z.Y.; Zhou, Z.X.; Sun, M.G.; Babicheva, V.E.; Chen, K.P. Nonradiating Silicon Nanoantenna Metasurfaces as Narrowband Absorbers. ACS Photonics 2018, 5, 2596-2601. [CrossRef]

24. Liu, C.; Wu, T.; Liu, Y.; Li, J.; Wang, Y.; Yu, Z.; Ye, H.; Yu, L. Realization of perfect selective absorber based on multipole modes in all-dielectric moth-eye structure. Opt. Express 2019, 27, 5703. [CrossRef] [PubMed]

25. Li, C.; Fan, H.; Dai, Q.; Wei, Z.; Lan, S.; Liu, H. Multipole Resonance in Arrays of Diamond Dielectric: A Metamaterial Perfect Absorber in the Visible Regime. Nanomaterials 2019, 9, 1222. [CrossRef] [PubMed]

26. Evlyukhin, A.B.; Reinhardt, C.; Seidel, A.; Luk'yanchuk, B.S.; Chichkov, B.N. Optical response features of Si-nanoparticle arrays. Phys. Rev. B 2010, 82, 045404. [CrossRef]

(C) 2020 by the authors. Licensee MDPI, Basel, Switzerland. This article is an open access article distributed under the terms and conditions of the Creative Commons Attribution (CC BY) license (http:/ / creativecommons.org/licenses/by/4.0/). 